\title{
Cross-Individual Gesture Recognition Based on Long Short-Term Memory Networks
}

\author{
Huasong Min $\mathbb{D}^{1},{ }^{1}$ Ziming Chen $\left(\mathbb{D},{ }^{1}\right.$ Bin Fang $\left(\mathbb{D},{ }^{2}\right.$ Ziwei Xia $\left(\mathbb{D},{ }^{3}\right.$ Yixu Song, ${ }^{2}$ Zongtao Wang, \\ Quan Zhou, ${ }^{5}$ Fuchun Sun $\mathbb{D D}^{2}$ and Chunfang Liu' \\ ${ }^{1}$ Laboratory for Embedded System and Intelligent Robot, Wuhan University of Science and Technology, Wuhan 430000, China \\ ${ }^{2}$ Department of Computer Science and Technology, Tsinghua University, Beijing 100084, China \\ ${ }^{3}$ School of Engineering and Technology, China University of Geoscience (Beijing), Beijing 100083, China \\ ${ }^{4}$ Key Lab of Industrial Computer Control Engineering of Hebei Province, Yanshan University, Qinghuangdao 066000, China \\ ${ }^{5}$ Anhui Province Key Laboratory of Special Heavy Load Robot, Anhui University of Technology, Ma'anshan 243000, China \\ ${ }^{6}$ Faculty of Information and Technology, Beijing University of Technology, Beijing 100124, China
}

Correspondence should be addressed to Bin Fang; fangbin@mail.tsinghua.edu.cn

Received 8 December 2020; Revised 26 May 2021; Accepted 29 June 2021; Published 8 July 2021

Academic Editor: Antonio J. Peña

Copyright ( $(2021$ Huasong Min et al. This is an open access article distributed under the Creative Commons Attribution License, which permits unrestricted use, distribution, and reproduction in any medium, provided the original work is properly cited.

Gestures recognition based on surface electromyography (sEMG) has been widely used for human-computer interaction. However, there are few research studies on overcoming the influence of physiological factors among different individuals. In this paper, a cross-individual gesture recognition method based on long short-term memory (LSTM) networks is proposed, named cross-individual LSTM (CI-LSTM). CI-LSTM has a dual-network structure, including a gesture recognition module and an individual recognition module. By designing the loss function, the individual information recognition module assists the gesture recognition module to train, which tends to orthogonalize the gesture features and individual features to minimize the impact of individual information differences on gesture recognition. Through cross-individual gesture recognition experiments, it is verified that compared with other selected algorithm models, the recognition accuracy obtained by using the CI-LSTM model can be improved by an average of $9.15 \%$. Compared with other models, CI-LSTM can overcome the influence of individual characteristics and complete the task of cross-individual hand gestures recognition. Based on the proposed model, online control of the prosthetic hand is realized.

\section{Introduction}

The core task of sEMG-based prosthetic hand control and human-computer interaction applications is to decode human motion intentions through sEMG signals [1]. $\mathrm{Hu}-$ man motions can be regarded as a combination of discrete and continuous motions [2]. Therefore, the research field of motion recognition based on sEMG is generally divided into two types of research questions. One is the research on identifying discrete motion states of limbs through sEMG signals, such as independent motion states like flexion and extension of fingers, making fists, and turning wrists [3-5]. And the other is research on using sEMG signals to estimate changes in joint continuous motion, such as joint torque and joint angle [6].

This paper only conducts research on the recognition of discrete movements of limbs through sEMG signals. Discrete action pattern classification is currently the most mature and fruitful method in the field of human action recognition based on sEMG. Representative research work includes the following: Englehart et al. [7] compared the effects of different features of sEMG on the accuracy of gesture classification and used linear discriminant analysis (LDA) for the first time to perform action recognition on the time-frequency domain features of sEMG, which can accurately identify 6 types of hand/wrist movements. Huang 
et al. [8] proposed to use a cascaded neural network with a feature self-organizing mapping mechanism to recognize 8 grasping movements, which improves the classification accuracy and performs better than models such as K nearest neighbours and back propagation neural network (BPNN). Chan and Englehart et al. [9] proposed using the hidden Markov model to recognize 6 upper limb movements, with a recognition accuracy of $94.6 \%$. Liu et al. [10] proposed generalized discriminant analysis combined with support vector machine (SVM) to construct a new classifier to achieve fast and high-precision classification of sEMG, and the recognition accuracy can reach $92 \%$. Chu et al. [11] proposed to use LDA combined with multilayer perceptron to recognize 9 kinds of hand/wrist movements based on the exploration of efficient feature projection methods and realize the online control of prosthetic hands.

Although the research results of discrete action classification are fruitful, it does not distinguish between personal habits and physiological differences of the experimental subjects, which will lead to greater randomness in the experimental results. Although the abovementioned research work has achieved relatively ideal accuracy, they are all based on the same individual data as the training set and the test set. The main focus is on designing a suitable classification model and selecting a feature set for classification. In the research work of gesture recognition investigated in this paper, none of them noticed that individual information such as behavior habits and exercise level may have a negative impact on gesture recognition. The composition of human hand muscles is basically the same, but the degree of development of muscle tissue is different between different subjects $[12,13]$. Therefore, the collected sEMG signals will also be different, which will naturally have a certain negative impact on gesture recognition. Based on this analysis, this article proposes related research ideas that train the classifier through the data of several individuals doing the same gestures and then uses the data that are not included in the training dataset for testing the performance of the classifier. This paper is based on this analysis to carry out cross-individual gesture recognition research.

Niu et al. [14] proposed a dual-network structure model, which can reduce the negative impact of individual shape information on the detection of facial action units by simultaneously learning the local relationship information of the face and the individual-specific shape information and orthogonalizing it. Inspired by the research work of paper [14], the key to realize the cross-individual gesture recognition is that the gesture recognition model needs to learn gesture features and individual information features at the same time and perform orthogonalization. Based on this idea, this paper proposes the cross-individual long shortterm memory (CI-LSTM) model based on long short-term memory (LSTM). To minimize the negative impact of different individual information on the accuracy of gesture recognition, CI-LSTM orthogonalizes gesture features and individual information features by learning gesture labels and individual labels at the same time. Furthermore, this paper realizes the online control of prosthetic hands based on CI-LSTM.

\section{Methods}

2.1. CI-LSTM. Learning gesture labels and individual labels at the same time is impossible for the traditional single network structure model because the single network structure model can only learn one type of feature at a time. Based on this analysis, in order to complete the task of crossindividual gesture recognition, this paper designs CI-LSTM, which is a dual-network structure model based on LSTM.

2.1.1. Model Structure. Gesture recognition based on sEMG can be naturally defined as a pattern classification problem, in which the classifier is usually trained through supervised learning. It is generally believed that the instantaneous value of the EMG signal is useless for gesture recognition [15-17]. This view is based on an empirical assumption because the original EMG signal of each channel is nonstationary, nonlinear, random, and unpredictable [18-20]. LSTM can remember the information in the past time through the gating mechanism and is recognized as good at processing time series signals [21]. Therefore, the CI-LSTM model we proposed is designed based on the LSTM model. In a neural network, long-term memory can be regarded as a network parameter, which implies characteristic information in the data, and its update cycle is much shorter than that of shortterm memory. In LSTM, the memory unit can capture key information at a certain moment and has the ability to store this key information for a certain time interval. The period of storing information in the memory unit is longer than shortterm memory, but it is much shorter than long-term memory. Therefore, it is called long short-term memory [22]. The LSTM network structure diagram is shown in Figure 1(a). The sEMG signal is essentially time series data [23], so CI-LSTM is based on the LSTM model that is good at processing time series signals.

The CI-LSTM model has a dual-network structure, which are a gesture recognition module and an individual information recognition module. Both modules contain an LSTM network, a hidden layer, and a fully connected (FC) layer. The overall structure diagram of CI-LSTM is shown in Figure 1(b).

In the offline training phase, the signals input to the two modules are the same, but the gesture recognition module only learns the labels of gestures, and the individual recognition module only learns the labels of individual information. The hidden layer of the gesture recognition module is only responsible to extract the features related to gesture recognition, regardless of the classification of individual information, then input the extracted features into the fully connected layer, and output the probability model of gesture categories $H=\left\{h_{1}, h_{2}, h_{3}, h_{4}, \ldots, h_{m}\right\}$. The hidden layer of the individual recognition module is only responsible for extracting the features of individual information, regardless of the classification of gestures, and then the extracted features are input to the fully connected layer, and the probability models of individual categories $P=\left\{p_{1}, p_{2}, p_{3}, \ldots, p_{n}\right\}$ are output. Among them, only the probability model $H=\left\{h_{1}, h_{2}, h_{3}, h_{4}, \ldots, h_{m}\right\}$ participated in 


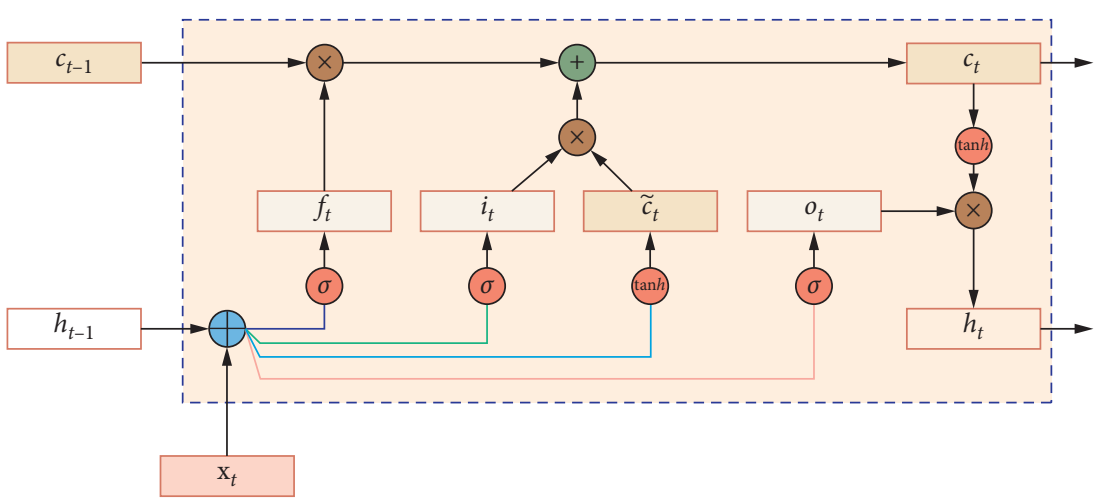

(a)

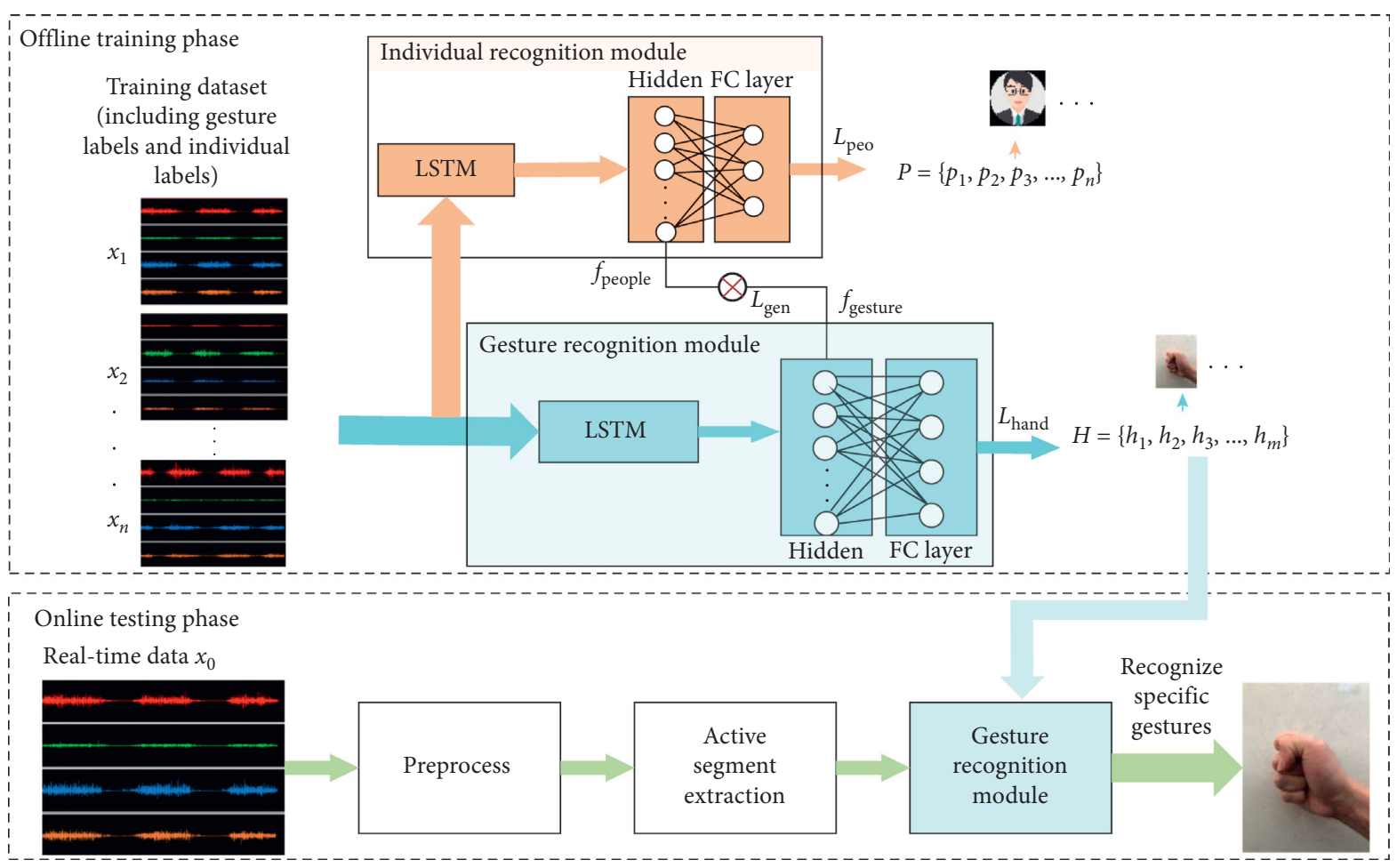

(b)

Figure 1: (a) Standard LSTM schematic diagram. (b) The overall structure of CI-LSTM.

the online testing phase when the probability model $P=$ $\left\{p_{1}, p_{2}, p_{3}, \ldots, p_{n}\right\}$ only assist the training of the gesture recognition module in the offline training phase. The main function of the individual probability model is to enable the individual recognition module to extract the characteristics of the individual information better and not as the output of model.

2.1.2. Realization of Cross-Individual Function. To minimize the negative impact of individual information differences on the accuracy of gesture recognition, this paper implements the cross-individual function of the CI-LSTM model by designing its loss function; that is, based on the principle of cosine similarity, the orthogonality of gesture features and individual information features is achieved through inner product calculations.
The principle of cosine similarity is to evaluate the similarity of two vectors by calculating the cosine of the angle between them [24]. Therefore, the angle can be measured to judge the similarity of the vectors. Anyway, the smaller the angle between two vectors, the more similar they are. The cosine similarity formula is shown as follows:

$$
\cos (u, v)=\frac{\sum_{i=1}^{k} u_{i} v_{i}}{\sqrt{\left(\sum_{i=1}^{k} u_{i}^{2}\right)} \sqrt{\left(\sum_{i=1}^{k} v_{i}^{2}\right)}}
$$

where $u_{i}$ and $v_{i}$ are the vectors of cosine similarity we require.

In the actual calculation process, calculating the inner product between two vectors can be approximately regarded as a simplified calculation of the cosine similarity between two vectors. And this paper uses this idea to realize the crossindividual function of the CI-LSTM model. Computing the 
absolute value of the inner product between the hidden layer state vector of the gesture recognition module and the individual recognition module is defined as the generalization loss $L_{\text {gen }}$, which is a part of the loss function of the CI-LSTM. The calculation formula is shown as follows:

$$
L_{\text {gen }}=\left|f_{\text {gesture }} \odot f_{\text {people }}\right|,
$$

where $f_{\text {gesture }}$ is the feature extracted by gesture recognition module, $f_{\text {people }}$ is the feature extracted by individual recognition module, and $\odot$ represents the inner product of two vectors.

The loss function of CI-LSTM should be defined consisting of three parts, including the loss of gesture classification $L_{\text {hand }}$ obtained by the gesture recognition module, the generalization loss $L_{\text {gen }}$ mentioned in the previous section, and the loss of individual information classification $L_{\text {peo }}$ obtained by individual recognition module. The loss function formula is shown as follows:

$$
L_{\text {total }}=\mu L_{\text {hand }}+\lambda L_{\text {gen }}+\omega L_{\text {peo }},
$$

where $\mu, \lambda$, and $\omega$ are the balance hyperparameters.

In the process of model iteration, the total loss will continue to decrease, which means that $L_{\text {gen }}$ is also continuously decreasing. According to the principle of cosine similarity, this indicates that the similarity of the feature vectors extracted from two modules is reduced; that is, the extracted feature vector from the gesture recognition module has a stronger correlation with the gesture itself, and the feature vector extracted by the individual recognition module is more related to individual information.

The goal of this design is to realize the cross-individual gesture recognition function of the CI-LSTM. The gesture characteristics learned by gesture recognition module and the individual information characteristics learned by the individual recognition module tend to be orthogonal so as to reduce the negative impact of information differences on the accuracy of gesture recognition as much as possible.

2.2. Experimental Design. We selected four gestures including fist, spread, thumb and index finger side pinch (TISP), and pinch with three fingers (PWTFs). Among them, fist and spread are the most basic gesture, TISP involves holding small pieces of objects such as keys and cards in daily life, and PWTF involves holding most of the smaller objects (see Figure 2). The sEMG sensors were placed in four positions of the forearm of the participant's preferred hand, which are flexor carpi radialis (FCR), flexor digitorum superficialis (FDS), extensor carpi radialis (ECR), and extensor digitorum communis (EDC), as shown in Figure 3. At the same time, the individuals participating in the collection experiment all adopted the same fixed arm posture, alternately performing designated actions and maintaining a resting state for 2 seconds between each action. We adopted this to reduce the influence of other factors on the experiment.

2.3. Active Segment Extraction. To improve the recognition accuracy of gestures, active segment extraction is necessary after the input signal is preprocessed. The active segment of sEMG can straightforwardly describe the corresponding signal changes during a single muscle movement; that is, the collected signal is divided into a forceful motion signal segment and a nonforced resting signal segment to determine the start and the end of each gesture. After recent years of research, there are a large number of mature active segment extraction algorithms for references, such as moving average method, short-time Fourier method, and entropy-based method. Considering that real-time and high efficiency are critical to the user experience of prosthetic hands, this paper chooses the simple and effective moving window method based on threshold judgment to detect the active segment and label the corresponding gesture on the active segment. Firstly, set the corresponding threshold value for the preprocessed sEMG signal. According to the statistics of scientific methods, the threshold value is generally set to $30 \%$ of the maximum amplitude in the set of experimental data, which is used to find the starting point and ending point of all active segments. Because there may be continuous contraction of muscles between motion segment and resting segment, it is also necessary to calculate the distance between each set of starting point and ending point. Through conversion, the starting point and ending point with an interval greater than $1.5 \mathrm{~s}$ are regarded as valid points so that all the data between the valid points can be marked with corresponding gesture labels. The extraction experiments of FIST show that the selected method based on threshold judgment works well (see Figure 4).

2.4. Assessment Indicators. To prove the advantages of the classification model we proposed, we have selected a series of evaluation indicators as standards for comparison with other algorithms, including Accuracy, Recall, and F1 Score. The expressions are as follows:

$$
\begin{aligned}
\text { Accuracy } & =\frac{\mathrm{TP}+\mathrm{TN}}{\mathrm{TP}+\mathrm{TN}+\mathrm{FP}+\mathrm{FN}}, \\
\text { Recall } & =\frac{\mathrm{TP}}{\mathrm{TP}+\mathrm{FN}}, \\
\text { Pre } & =\frac{\mathrm{TP}}{\mathrm{TP}+\mathrm{FP}}, \\
\text { F1_Score } & =\frac{2 * \text { Pre } * \text { Recall }}{\text { Pre }+ \text { Recall }},
\end{aligned}
$$

where TP (True Positive) is samples of positive that are correctly recognized by the model. FP (False Positive) is samples of negative that are recognized as positive by the model. TN (True Negative) is samples of negative that are correctly recognized by the model. FN (False Negative) is samples of positive that are recognized as negative by the model.

\section{Results and Discussion}

3.1. Participants and Cross-Individual Dataset. We have selected 4 healthy men aged 25-27 with different exercise 


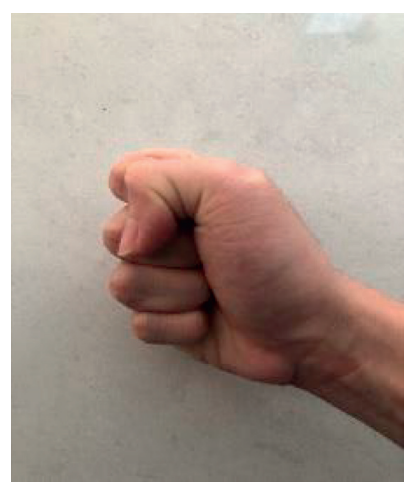

(a)

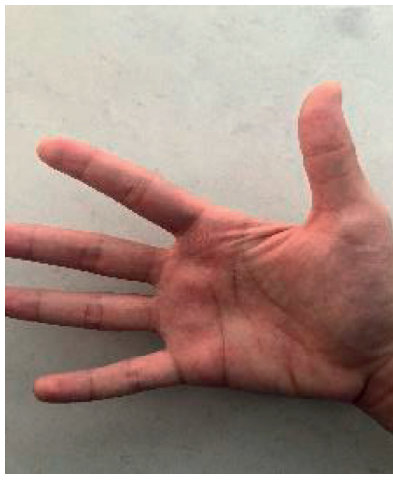

(b)

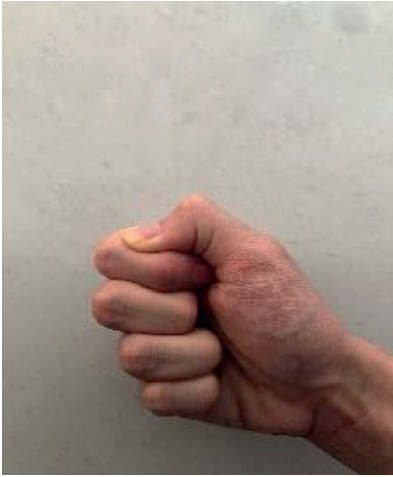

(c)

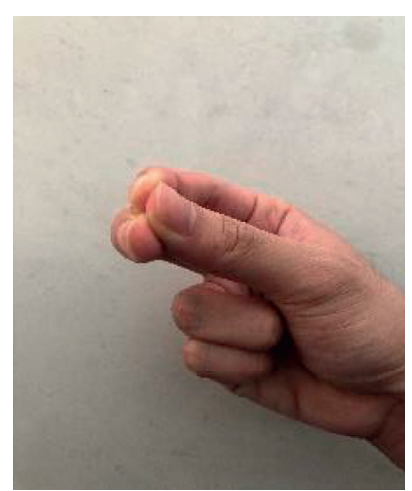

(d)

FiguRe 2: The selected gestures for experiment: (a) fist; (b) spread; (c) thumb and index finger side pinch; (d) pinch with three fingers.

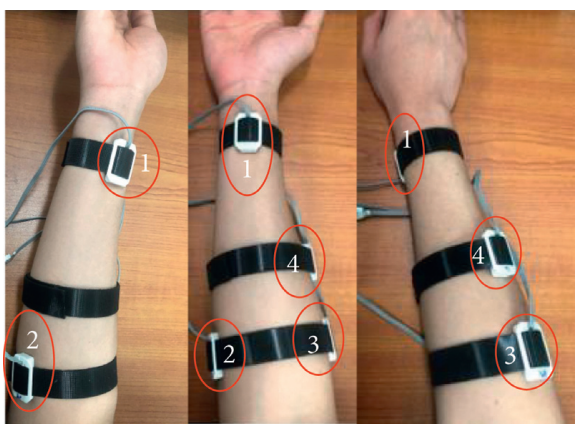

FIGURE 3: Schematic diagram of sEMG sensors placement (number 1 is FCR area, number 2 is FDS area, number 3 is ECR area, and number 4 is EDC area).

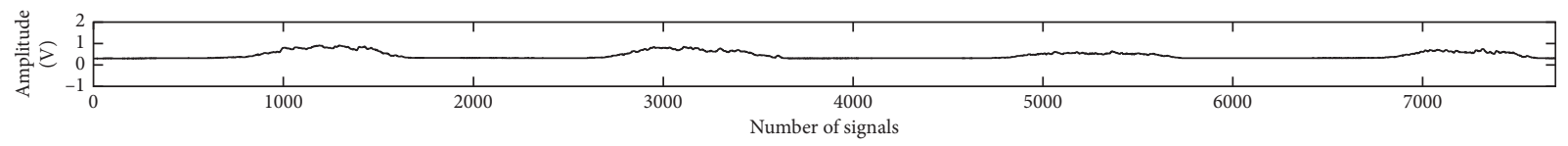

(a)

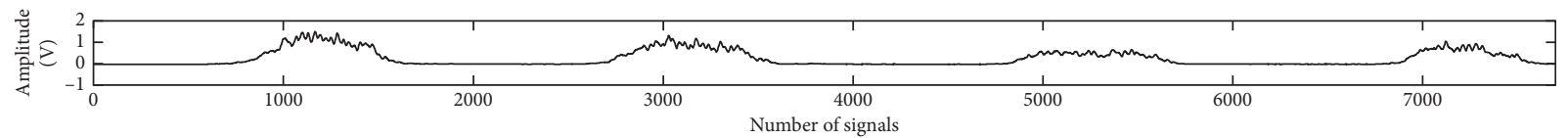

(b)

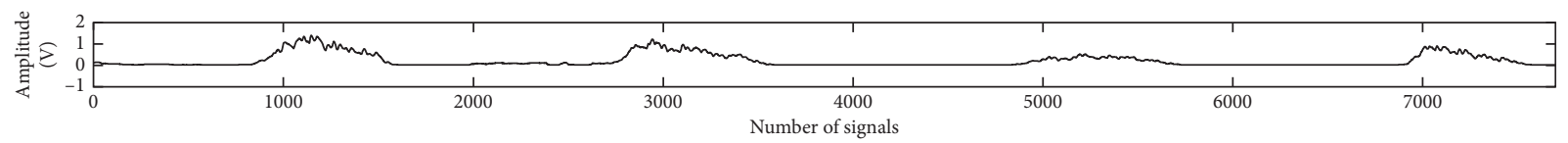

(c)

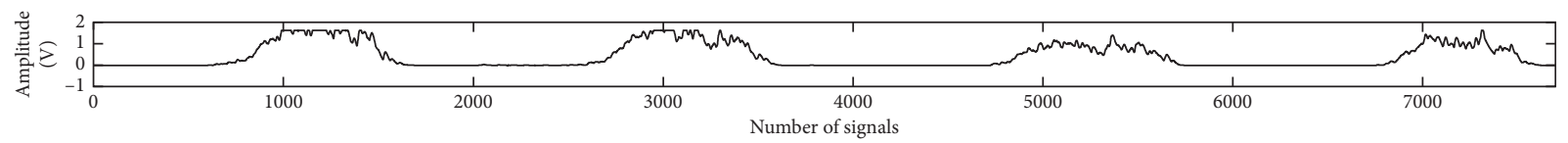

(d)

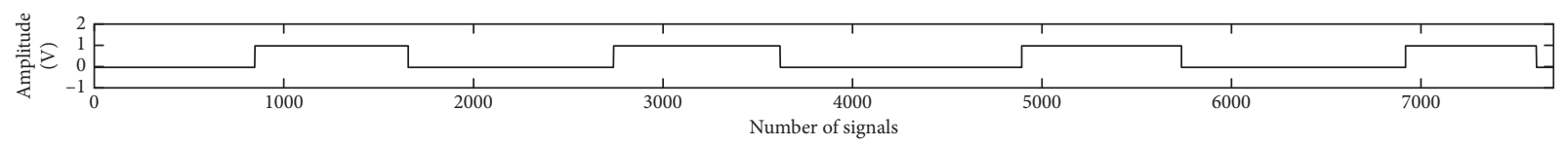

(e)

FIGURE 4: Effect diagram of active segment extraction (fist, corresponding label is 1): (a) sensor 1; (b) sensor 2; (c) sensor 3; (d) sensor 4; (e) Label. 
levels and behavior habits as the participants in this paper, hereinafter referred to as subjects A, B, C, and D. In order to verify the reliability of the algorithm, the training set is composed of the data of 3 participants, and the test set is the data of the remaining participant, named as four combinations of ABC_D, ABD_C, ACD_B, and BCD_A (see Table 1). Firstly, the original sEMG signal collected from 4 subjects is preprocessed by the bandpass filter of $10 \mathrm{~Hz}-300 \mathrm{~Hz}$ and the notch filter of $50 \mathrm{~Hz}$, and then the active segment of the signal is extracted according to $30 \%$ of the maximum amplitude of each data as the threshold. The obtained data are divided into $80 \%$ training set, $10 \%$ validation set, and $10 \%$ test set. The corresponding data are combined according to the grouping method, and the cross-individual experimental dataset is designed. Due to different daily habits, there will inevitably be an imbalance in the number of data obtained when different individuals perform the same gesture, resulting in an imbalance in the data of the training set. This is the most common problem in training sets [25]. Because the collected sEMG signal is a time series signal, we choose the undersampling method to solve the category imbalance in the training set. The sample distribution of each group is shown in Figure 5.

\subsection{Cross-Individual Gesture Recognition Experiment.} This paper pays more attention to verifying the cross-individual gesture recognition ability of CI-LSTM, so the gesture recognition performance is the key, and the classification loss of individual information $L_{\text {peo }}$ is used as the weight regularization item of the proposed model. The recognition performance of gesture actions is the key, so the hyperparameter $\mu=1$. The hyperparameters $\lambda$ and $\omega$ are used to balance the overall loss function value of CI-LSTM. This paper chooses the hyperparameters as $\mu=1, \lambda=0.8$, and $\omega=0.2$ based on experience.

To prove the effectiveness of our proposed model, we used SVM, standard LSTM, CNN-LSTM, and CI-LSTM to compare the classification effects of the four datasets (see Table 2). Among them, the model structure of CNN-LSTM is similar to CI-LSTM, but the individual recognition module of CI-LSTM is replaced with CNN. Table 3 shows the indicators of the experimental group with the best performance of the four models mentioned in this paper for individual generalization experimental data. In the experiments of individual generalized hand gesture recognition with different data combinations, the model we proposed shows better recognition results than other models. Compared with a single network structure, the accuracy rate is improved on average $11.88 \%$, and the highest can reach $32.71 \%$; compared with the CNN-LSTM model with the same dual-network structure, it also has a maximum improvement of $6.87 \%$.

In cross-individual gesture recognition experiments with the cross-individual dataset, CI-LSTM shows a better recognition effect than other models. CI-LSTM can achieve an average accuracy of $70.58 \%$ when faced with test data not included in the training set. Based on the above experiments,
TABLE 1: Group and name of the participant.

\begin{tabular}{lcc}
\hline Training set & Testing set & Name \\
\hline A, B, C & D & ABC_D \\
A, B, D & C & ABD_C \\
A, C, D & B & ACD_B \\
B, C, D & A & BCD_A \\
\hline
\end{tabular}

it is verified that the CI-LSTM model can orthogonalize the gesture characteristics and the individual information characteristics through the design of the loss function and reduce the negative impact of different individual information on gesture recognition accuracy as much as possible. Comparing with other algorithms in this paper, CI-LSTM can effectively improve the accuracy of cross-individual gesture recognition.

According to the experimental results of group BCD_A (see Table 3) and the confusion matrix of each model (see Figure 6), CI-LSTM not only has the highest average recognition accuracy but also has an improvement of $6.87 \%$ compared with CNN-LSTM. Compared with CNN-LSTM and LSTM, CI-LSTM is more sensitive to the two similar gestures of FIST and TISP and the recognition accuracy of FIST can be increased by $16 \%-24 \%$. Although the recall of FIST is not as good as the performance of SVM, the average accuracy is far better than SVM. This may also benefit from the design of the dual-network structure and loss function. By extracting features that are more related to gesture itself, the recognition accuracy of the gesture that is easy to be misjudged can be improved.

3.3. Online Control Experiment of Prosthetic Hand. The complete process of the prosthetic hand online control includes offline training module and online recognition module (see Figure 7). The offline training module is for classifier training. First step of offline training module is to collect the sEMG of the selected subjects. The experiments in this section are based on the four movements of FIST, SPREAD, TISP, and PWTF. Each subject is collected 90 times for each gesture. After preprocessing $[26,27]$ and extracting active segment by the method in Section 2.3, the obtained data are divided into $80 \%$ as the training set, $10 \%$ as the validation set, and $10 \%$ as the test set to create a dataset to train the model.

The online recognition module is for reproducing gestures. After preprocessing and extracting active segment, the sEMG collected in real time is input to the trained model of the offline training module to obtain the classification result and output the label corresponding to specific gesture. According to the obtained label, the corresponding control instruction is sent to the prosthetic hand control system, and the recognized gesture is reproduced by the prosthetic hand. The prosthetic hand selected in this paper can make all the selected movements (see Figure 8).

We take the online control effect of the fist recognition as an example for analysis as an example (see Figure 9). Firstly, the subject makes a fist, the sensors placed on the forearm collect sEMG signal at this time, and the signal acquisition 


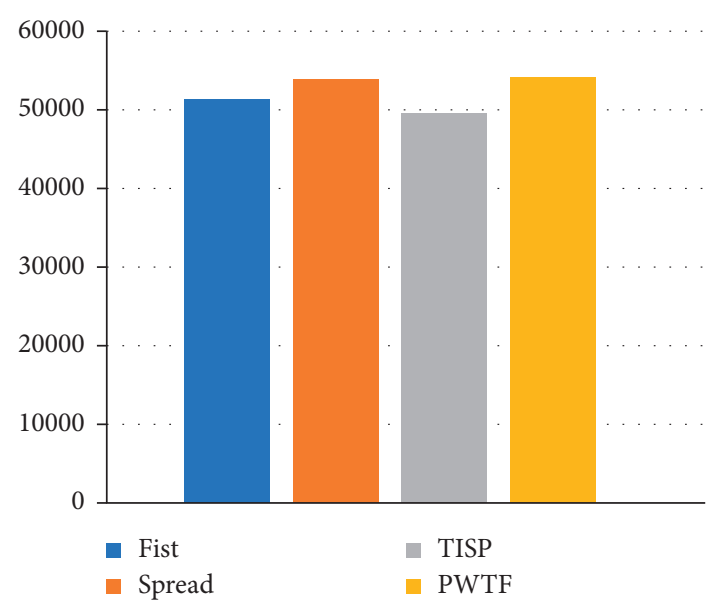

(a)

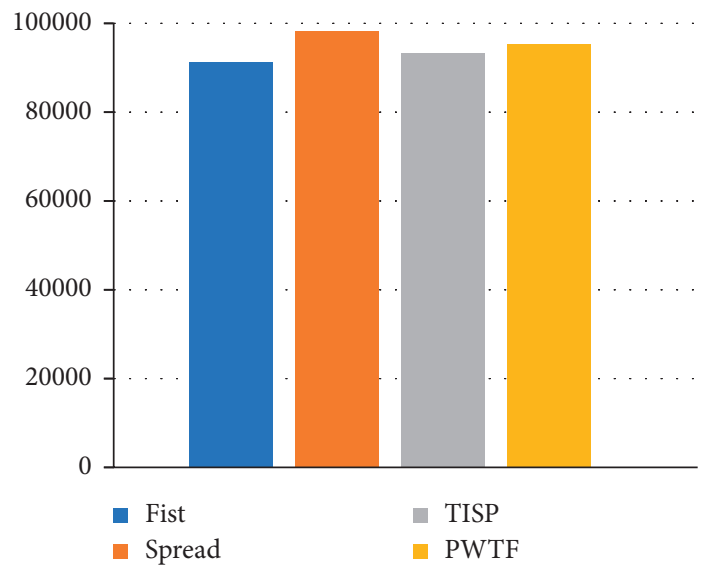

(c)

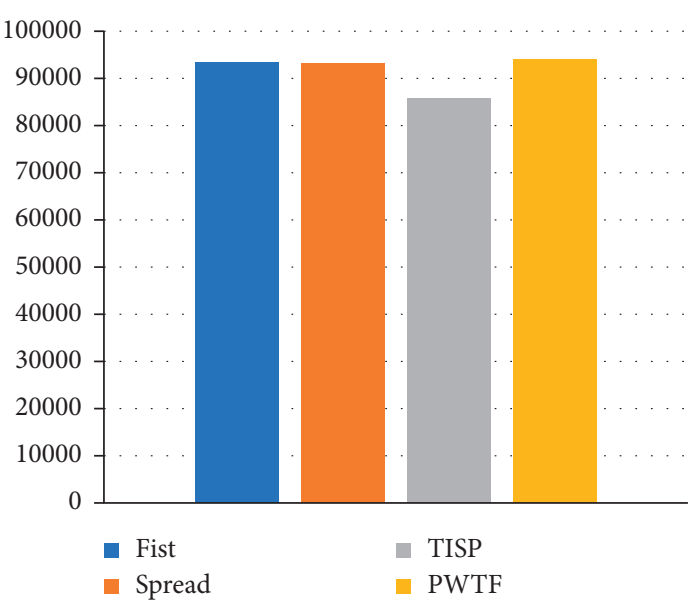

(b)

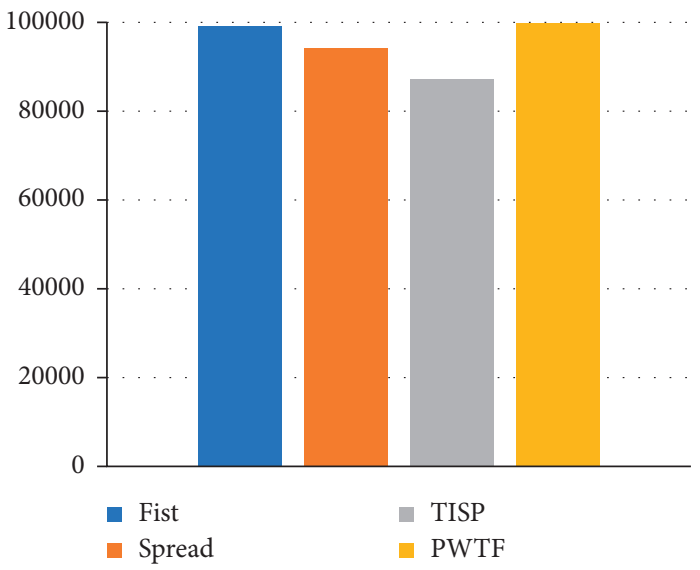

(d)

FIgUre 5: Sample distribution cross-individual dataset: (a) ABC_D; (b) ABD_C; (c) ACD_B; (d) BCD_A.

TABLE 2: Comparison table of four datasets of each model.

\begin{tabular}{lcc}
\hline Group & Model & Average accuracy (\%) \\
\hline & CI-LSTM & $\mathbf{7 6 . 4 6}$ \\
ABC_D & CNN-LSTM & 72.32 \\
& LSTM & 68.63 \\
& SVM & 43.75 \\
\hline \multirow{2}{*}{ ABD_C } & CI-LSTM & $\mathbf{6 3 . 0 9}$ \\
& CNN-LSTM & 58.09 \\
& LSTM & 59.04 \\
& SVM & 55.00 \\
\hline ACD_B & CI-LSTM & $\mathbf{6 5 . 7 5}$ \\
& CNN-LSTM & 65.63 \\
& LSTM & 64.18 \\
& SVM & 61.88 \\
\hline BCD_A & CI-LSTM & $\mathbf{7 7 . 0 0}$ \\
& CNN-LSTM & 70.13 \\
& LSTM & 67.87 \\
\hline
\end{tabular}

microcontroller is responsible for receiving the signal collected by the sensor and sending it to PC in real time. After receiving the data, $\mathrm{PC}$ preprocesses it and extracts the active segment. Then, the processed data are input into the CI-
TABLE 3: Group BCD_A assessment indicators.

\begin{tabular}{lccccc}
\hline Model & Gestures & $\begin{array}{c}\text { Recall } \\
(\%)\end{array}$ & $\begin{array}{c}\text { F1_score } \\
(\%)\end{array}$ & Support & $\begin{array}{c}\text { Accuracy } \\
(\%)\end{array}$ \\
\hline \multirow{4}{*}{ CI- } & Fist & 40 & 54 & 5305 & \\
LSTM & Spread & 97 & 91 & 6058 & 77.00 \\
& TISP & 83 & 72 & 4432 & \\
& PWTF & 86 & 82 & 5601 & \\
CNN- & Fist & 24 & 35 & 5305 & \\
LSTM & Spread & 92 & 89 & 6058 & 70.13 \\
& TISP & 80 & 63 & 4432 & \\
& PWTF & 83 & 80 & 5601 & \\
LSTM & Fist & 16 & 26 & 5305 & \\
& Spread & 92 & 91 & 6058 & 67.87 \\
& TISP & 83 & 61 & 4432 & \\
\hline \multirow{4}{*}{ SVM } & PWTF & 79 & 74 & 5601 & \\
& Fist & 60 & 49 & 5305 & \\
& Spread & 78 & 62 & 6058 & 50.63 \\
\hline
\end{tabular}

LSTM model trained offline based on the BCD_A grouped data in Section 3.2 to recognize the fist gesture and then output the label corresponding to fist. According to the label, 


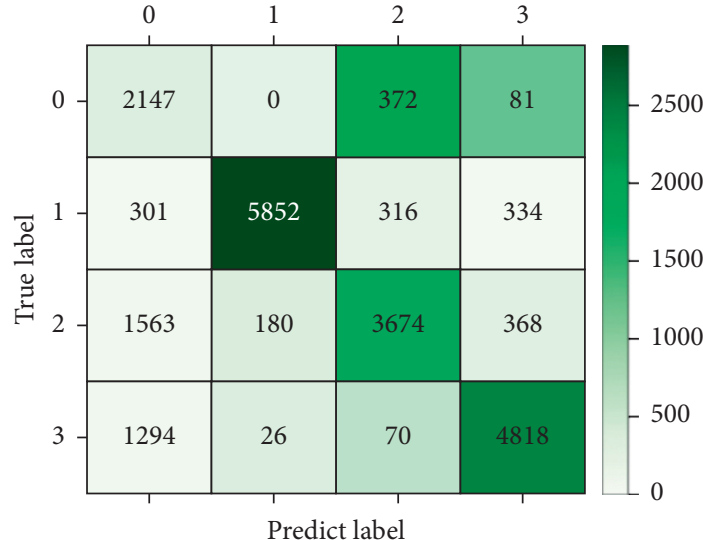

(a)

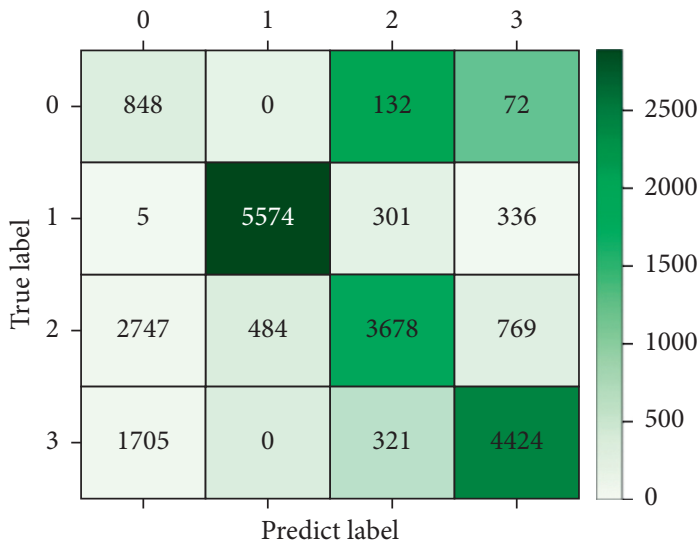

(c)

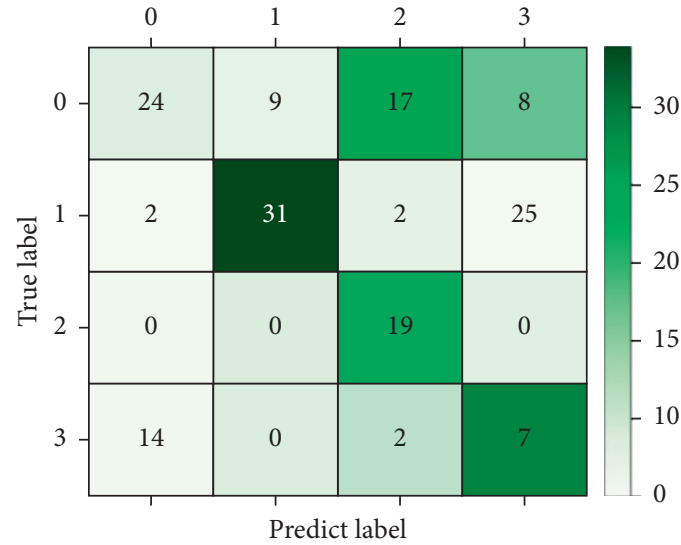

(b)

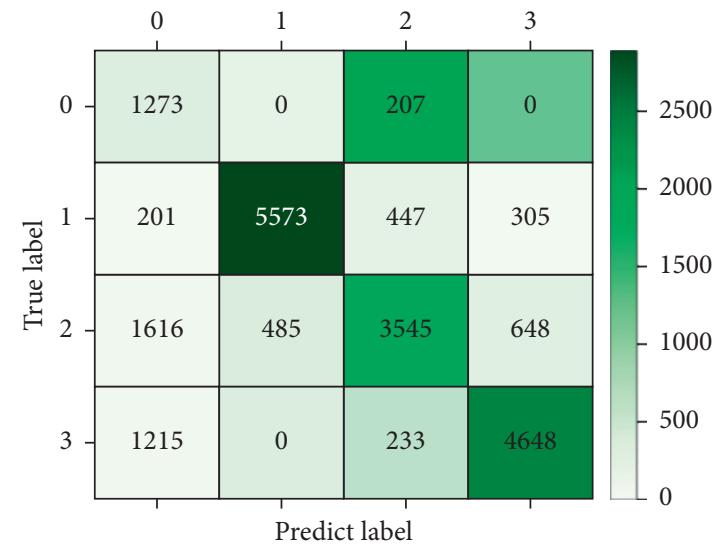

(d)

FIgure 6: Confusion matrix of each model: (a) CI-LSTM; (b) SVM; (c) LSTM; (d) CNN-LSTM.

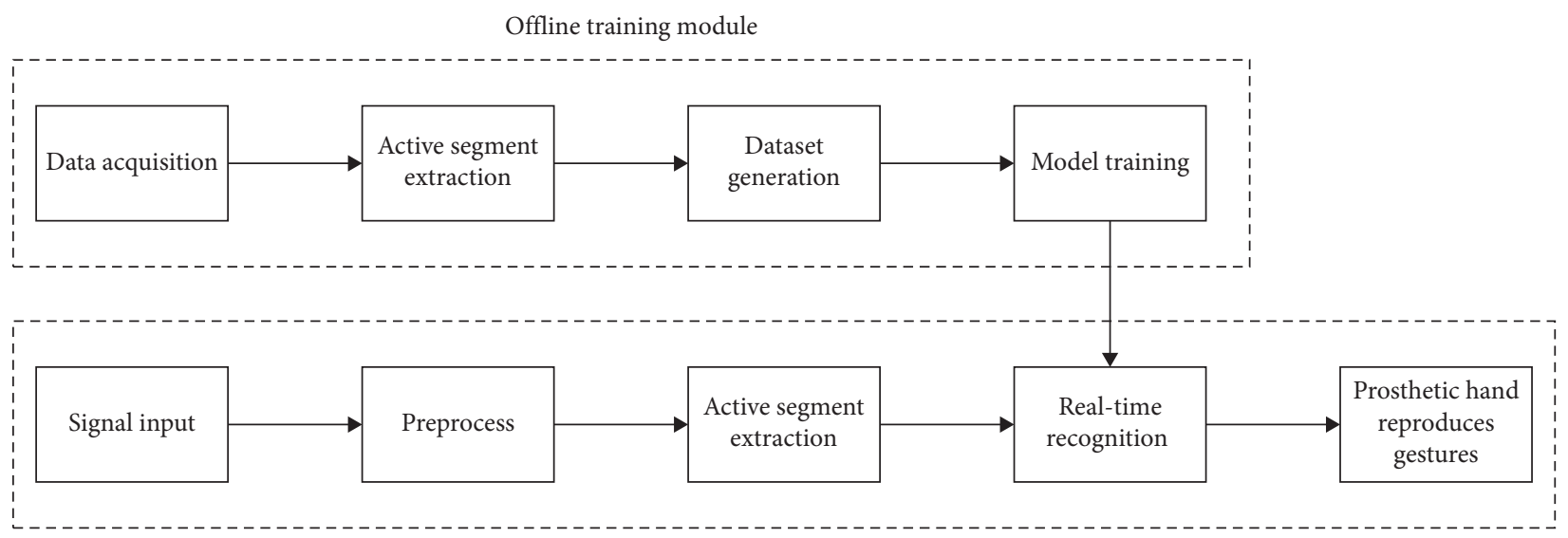

Online recognition module

FIGURE 7: Online recognition flow chart.

the corresponding motor rotation angle value is transmitted to the prosthetic hand control system, and the prosthetic hand reproduces the fist gesture.

According to the experimental results in Section 3.2, the average classification accuracy is $77 \%$. To improve the performance in the experiment, we adopt a voting decisionmaking method in the prosthetic hand online control system to reduce the influence of network model misclassification. To recognize the gesture more accurately at the current moment, it is not necessary to encode each classification result into a control instruction but to refer to the output results of the classification model at the previous or later moments at that moment. After that, we select the gesture with the most occurrences from the specified number of 

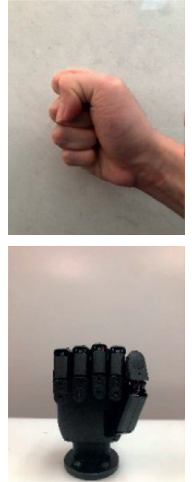

(a)
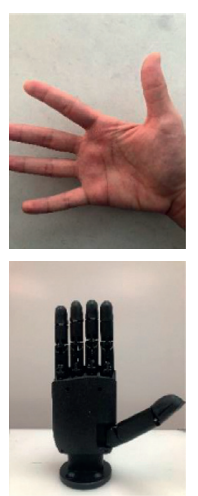

(b)
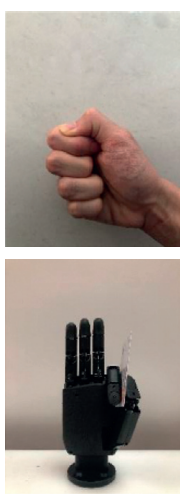

(c)
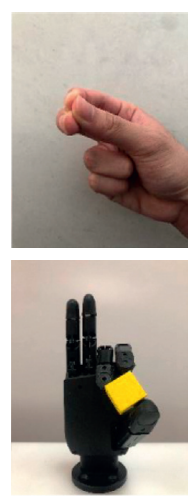

(d)

Figure 8: Prosthetic hand imitating human gestures: (a) fist; (b) spread; (c) TISP; (d) PWTF.

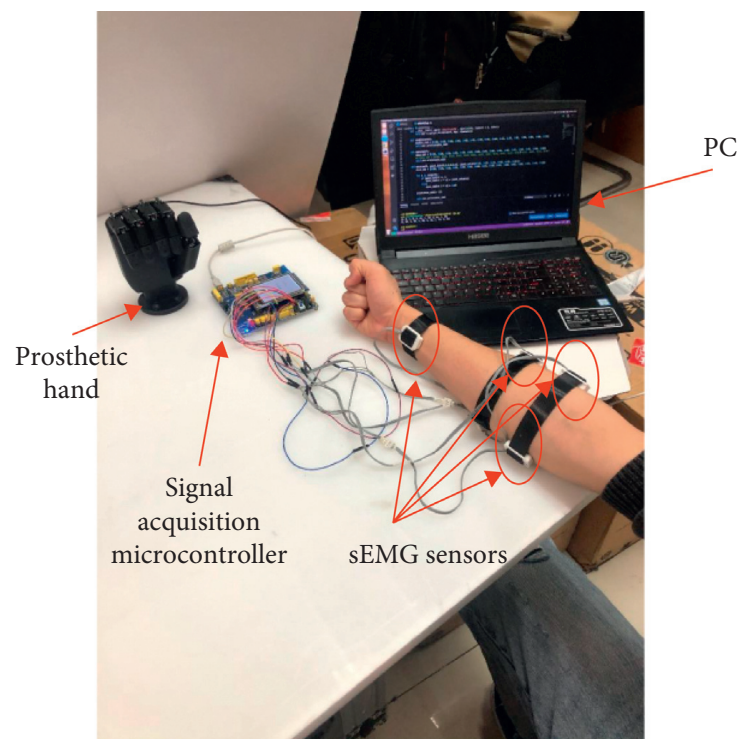

FIgURE 9: Online control experiment of prosthetic hand (fist).

TABLE 4: Online control experiment results.

\begin{tabular}{lccc}
\hline Gesture & Total number of executions & Correct number of executions & Accuracy $(\%)$ \\
\hline Fist & 50 & 32 & 64 \\
Spread & 50 & 49 & 98 \\
TISP & 50 & 45 & 90 \\
PWTF & 50 & 46 & 92 \\
\hline
\end{tabular}

classification results as the valid gesture at the current moment and then encode it for the prosthetic hand to reproduce the corresponding gesture. The results of the corresponding gestures reproduced by the prosthetic hand are shown in Table 4 . The average recognition accuracy after the introduction of the decision method is $86.5 \%$, which is $9.5 \%$ higher than the average recognition accuracy of the classification model.

3.4. Discussion. Instead of choosing hand gestures that are easier to distinguish from each other, we choose more meaningful and important gestures in human life for experiments even if they involve similar gestures. According to the experimental results, although CI-LSTM performs better for similar gestures compared with LSTM and CNN-LSTM, the accuracy is still not good enough. In our future work, more attention should be paid to improving the model's ability to recognize similar gestures.

In general, as long as the control period from the intention of the prosthetic hand user to the corresponding gesture of the prosthetic hand is controlled within $250 \mathrm{~ms}$ [28], the user will not feel the operation delay. Therefore, the online recognition speed of the prosthetic hand control 
system is certain requirements. After average time statistics, the prosthetic hand control system built in this article has not perfectly met the conditions of real-time response, and improvement work is still needed.

\section{Conclusion}

To overcome the negative impact of individual information differences such as behavior habits and exercise levels on gesture recognition, a cross-individual gesture recognition model CI-LSTM based on LSTM is proposed. CI-LSTM has a dual-network structure. By designing the loss function, the individual information recognition module assists the gesture recognition module to train, which tends to orthogonalize the gesture features and individual features to minimize the impact of individual information differences on gesture recognition. According to the experimental results, it is verified that CI-LSTM can effectively overcome the influence of individual characteristics and complete the task of cross-individual gesture recognition with a better accuracy. Although the accuracy of cross-individual gesture recognition tasks cannot reach the accuracy of existing gesture recognition research, we prove that differences in individual characteristics have a certain impact on gesture recognition. Meanwhile, we affirm the significance and importance of cross-individual gesture recognition.

\section{Data Availability}

The data used to support the findings of this study are supplied by Ziming Chen under license and so cannot be made freely available. Requests for access to these data should be made to Ziming Chen (ziming_chen1209@ 163.com).

\section{Conflicts of Interest}

The authors declare that they have no conflicts of interest.

\section{Authors' Contributions}

Huasong Min and Ziming Chen contributed equally to this work.

\section{Acknowledgments}

This work was jointly supported by the National Key R\&D Program of China (Grant no. 2019YFB1309800), National Natural Science Foundation of China (Grant nos. 91848206 and 61703230), and Beijing Science \& Technology Project (Grant no. Z191100008019008).

\section{References}

[1] C. J. Heckman and R. M. Enoka, "Motor unit," Comprehensive Physiology, vol. 2, no. 4, pp. 2629-2682, 2012.

[2] O. Cyrus, J. Mile, B. Timothy et al., "Policies for neural prosthetic control: initial experiments with a text interface," in Proceedings of the American Control Conference, vol. 7, pp. 4161-4166, Seattle, WA, USA, June 2008.
[3] L. Liao, Y. Tseng, H. Chiang, and W. Y. Wang, "EMG-based control scheme with SVM classifier for assistive robot arm," in Proceedings of the International Automatic Control Conference (CACS), pp. 1-5, IEEE, Taoyuan, Taiwan, November 2018.

[4] N. M. Krishnan, M. Mariappan, K. Muthukaruppan, M. H. A. Hijazi, and W. W. Kitt, "Electroencephalography (EEG) based control in assistive mobile robots: a review," in Proceedings of the IOP Conference Series: Materials Science and Engineering, IOP Publishing, Miri, Malasiya, November 2016.

[5] Q. Huang, Y. Chen, Z. Zhang et al., "An EOG-based wheelchair robotic arm system for assisting patients with severe spinal cord injuries," Journal of Neural Engineering, vol. 16, no. 2, Article ID 026021, 2019.

[6] Y. Nam, B. Koo, A. Cichocki et al., "GOM-face: GKP, EOG, and EMG-based multimodal interface with application to humanoid robot control," IEEE Transactions on Biomedical Engineering, vol. 61, pp. 453-462, 2014.

[7] K. Englehart, B. Hudgins, P. A. Parker et al., "A wavelet-based continuous classification scheme for multifunction myoelectric control," IEEE Transactions on Biomedical Engineering, vol. 48, no. 3, pp. 302-311, 2001.

[8] H. P. Huang, Y. H. Liu, L. W. Liu et al., "EMG classification for prehensile postures using cascaded architecture of neural networks with self-organizing maps," in Proceedings of the IEEE International Conference on Robotics and Automation, pp. 1497-1502, Taipei, Taiwan, September 2003.

[9] A. D. Chan and K. B. Englehart, "Continuous myoelectric control for powered prostheses using hidden Markov models," IEEE Transactions on Biomedical Engineering, vol. 52, no. 1, pp. 121-124, 2005.

[10] Y. H. Liu, H. P. Huang, and C. H. Weng, "Recognition of electromyographic signals using cascaded kernel learning machine," IEEE, vol. 12, no. 3, pp. 253-264, 2007.

[11] J. U. Chu, I. Moon, Y. J. Lee, S. K. Kim et al., "A supervised feature-projection-base real-time EMG pattern recognition for multifunction myoelectric hand control," IEEE, vol. 12, no. 3, pp. 282-290, 2007.

[12] A. Rezeika, M. Benda, P. Stawicki, F. Gembler, A. Saboor, and I. Volosyak, "30-targets hybrid BNCI speller based on SSVEP and EMG," in Proceedings of the IEEE International Conference on Systems, Man, and Cybernetics (SMC), pp. 153-158, Miyazaki, Japan, October 2018.

[13] F. Zhang, D. Yang, and T. Liu, "Gesture recognition based on EMG signal and gesture signal," Sensors and Microsystems, vol. 38, no. 7, pp. 46-49, 2019.

[14] X. Niu, H. Han, and S. Yang, "Local relationship learning with person-specific shape regularization for facial action unit detection," in Proceedings of the IEEE/CVF Conference on Computer Vision and Pattern Recognition (CVPR), pp. 11917-11926, IEEE, Los Angeles, CA, USA, June 2019.

[15] M. A. Oskoei and H. Hu, "Myoelectric control systems-a survey," Biomedical Signal Processing and Control, vol. 2, pp. 275-294, 2007.

[16] L. H. Smith, L. J. Hargrove, T. A. Kuikan, and B. A. Lock, "Determining the optimal window length for pattern recognition-based myoelectric control: balancing the competing effects of classification error and controller delay," IEEE Transactions on Neural Systems and Rehabilitation Engineering, vol. 19, pp. 186-192, 2011.

[17] D. Farina and R. Merletti, "Comparison of algorithms for estimation of EMG variables during voluntary isometric contraction," Journal of Electromyography and Kinesiology, vol. 10, pp. 337-349, 2000. 
[18] A. Goen and D. Tiwari, "Review of surface electromyogram signals: its analysis and applications," International Journal of Electrical, Electronics, Communication, Energy Science and Engineering, vol. 7, pp. 965-973, 2013.

[19] S. Salminger, A. Sturma, C. Hofer et al., "Long-term implant of intramuscular sensors and nerve transfers for wireless control of robotic arms in above-elbow amputees," Science Robotics, vol. 4, no. 32, Article ID eaaw6306, 2019.

[20] A. Mansoor, M. W. Usman, N. Jamil et al., "Deep learning algorithm for brain-computer interface," Scientific Programming, vol. 2020, Article ID 5762149, 12 pages, 2020.

[21] X. Yuan, L. Li, Y. Shardt et al., "Deep learning with spatiotemporal attention-based LSTM for industrial soft sensor model development," IEEE Transactions on Industrial Electronics, vol. 68, no. 5, pp. 4404-4414, 2020.

[22] X. Yuan, L. Li, and Y. Wang, "Nonlinear dynamic soft sensor modelling with supervised long short-term memory network," IEEE Transactions on Industrial Informatics, vol. 16, no. 5, pp. 3168-3176, 2020.

[23] Y. Sun, C. Xu, G. Li et al., "Intelligent human computer interaction based on non-redundant EMG signal," Alexandria Engineering Journal, vol. 59, no. 3, pp. 1149-1157, 2020.

[24] W. Anwar, I. Sarwar Bajwa, and S. Ramzan, "Design and implementation of a machine learning-based authorship identification model," Scientific Programming, vol. 2019, Article ID 9431073, 14 pages, 2019.

[25] Y. Liu, Li Xiang, X. Chen et al., "High-performance machine learning for large-scale data classification considering class imbalance," Scientific Programming, vol. 2020, Article ID 1953461, 16 pages, 2020.

[26] D. Jiang, G. Li, Y. Sun et al., "Gesture recognition based on skeletonization algorithm and CNN with ASL database," Multimedia Tools and Applications, vol. 78, no. 21, Article ID 29953, 2019.

[27] J. Qi, G. Jiang, G. Li et al., "Surface EMG hand gesture recognition system based on PCA and GRNN," Neural Computing and Applications, vol. 32, no. 10, pp. 6343-6351, 2020.

[28] Q. Gao, J. Liu, and Z. Ju, "Robust real-time hand detection and localization for space human-robot interaction based on deep learning," Neurocomputing, vol. 390, pp. 198-206, 2019. 